\title{
History of Astrophysics in Antarctica - A Brief Overview
}

Balthasar T. Indermuehle ${ }^{\dagger}$, Michael G. Burton ${ }^{\ddagger}$, Sarah T. Maddison ${ }^{\dagger}$

${ }^{\dagger}$ Swinburne University, Centre for Astrophysics and Supercomputing, Hawthorn, VIC 3122, Australia

$\ddagger$ University of New South Wales, School of Physics, Sydney, NSW 2052, Australia

On examining the historical development of astrophysical science at the bottom of the world from the early 20 th century until today we find three temporally overlapping eras of which each has a rather distinct beginning. These are the eras of Astrogeology, High Energy Astrophysics and Photon Astronomy. ${ }^{15}$

\section{Astrogeological Era (from 1912)}

In 1912, Mawson discovered the Adelie Land Meteorite. Russian geologists found several meteorites of unrelated morphological types in the Lazarev region in 1961, giving rise to the ablation zone theory. This was followed in 1969 by the first formal meteorite search programme. Thousands of meteorites have since been found in Antarctica, mostly from glacial ablation zones.

\section{High Energy Era (from mid 1950's)}

In 1955, the first Antarctic astrophysical project was initiated with a cosmic ray detector installed at McMurdo station. A detector was installed at the South Pole in 1964 and others were commissioned at several national research stations. Today, cosmic rays with energies above $50 \mathrm{TeV}$ are recorded by SPASE 2 which is situated on top of $A M A N D A$. AMANDA II is scheduled to start operation in 2003 and by 2006 , Ice Cube, a $1 \mathrm{~km}^{3}$ detector, will open up the $\mathrm{PeV}$ energy region where the Universe is opaque to high energy $\gamma$-rays from beyond our galaxy.

\section{Photon Astronomy Era (from 1970's)}

Optical astronomy was first employed at the South Pole in 1964 for site testing. In 1979, a continuous 120 hours of solar observations allowed hundreds of solar eigenmodes to be discovered. Infrared and CMB measurements require the best atmospheric windows, and site testing since 1994 has demonstrated this is provided from the Antarctic plateau. In 1988, the first CMB measurement from Terra Nova Bay produced a map at an angular scale of $1.3^{\circ}$. In 1992, the CMB was imaged with better resolution by Python, a $0.75 \mathrm{~m}$ telescope. It was replaced in 1997 by Viper, a $2.1 \mathrm{~m}$ off-axis telescope, to be complemented in 1998 by DASI. The first flight of the Boomerang balloon in 1998 provided much improved angular resolution data for a CMB map published in late 2002. AST/RO, a $1.7 \mathrm{~m}$ sub-mm telescope, has been mapping the Galactic plane in $\mathrm{CI}$ and CO since 1995. Between 1994 and 1999, SPIREX imaged the $2-5 \mu \mathrm{m}$ continuum and PAHs with the Grim and Abu cameras.

\footnotetext{
${ }^{15} \mathrm{~A}$ full version of this paper will be submitted to PASA. The poster is on the SPS2 web site.
} 